\title{
On Exhaustion of Domains
}

\author{
Buma L. Fridman \& DaOWei Ma
}

ABStRACT. We give examples of non-homogeneous bounded domains in $\mathbb{C}^{n}$ which can exhaust no other domains. And we prove that every bounded domain in $\mathbb{C}^{n}$ with $C^{2}$ boundary can exhaust the ball.

1. Introduction. Let $D, G$ be bounded domains in $\mathbb{C}^{n}, n \geq 1$. We say that $D$ can exhaust $G$ if for any compact $K \Subset G$ there exists a biholomorphic imbedding $f: D \rightarrow G$ such that $f(D) \supset K$. This is equivalent to the following: There exists a sequence $f_{k}: D \rightarrow G$ of biholomorphic imbeddings such that the sets $f_{k}(D) \rightarrow G$ as $k \rightarrow \infty$, i.e., for any $K \Subset G$ there exists a number $N$ that for $k \geq N, f_{k}(D) \supset K$. Below we call such a sequence $\left\{f_{k}\right\}$ an exhausting sequence. We use the notation $G \sim D$ to indicate that $G$ is biholomorphically equivalent to $D$. Obviously if $G_{1} \sim G_{2}$ and $G_{1}$ can be exhausted by $D$, then so can $G_{2}$; therefore for the question of exhaustion one can consider the whole class $\left\{G_{1}\right\}$ of domains biholomorphically equivalent to $G_{1}$. We now introduce the set $\mathrm{E}(D)$ of all classes $\{G\}$, where $G$ is a bounded domain in $\mathbb{C}^{n}$ that can be exhausted by $D$. Obviously $\mathrm{E}(D)$ is not empty: it always contains $\{D\}$. By $\# \mathrm{E}(D)$ we denote the number of elements (different classes) in $\mathrm{E}(D)$.

The problem of exhausting one domain by another has been considered in a number of papers $([2-7])$. In this paper we are considering only bounded domains. One of the important problems is the following one: describe the set $\mathrm{E}(D)$ for a specific $D$. It can be shown that if $D$ is strongly pseudoconvex, then $\# \mathrm{E}(D) \leq 2$ : if $\{G\} \in \mathrm{E}(D)$, then either $G \sim D$ or $G \sim B_{n}$ - the unit ball in $\mathbb{C}^{n}$ (see [5]). In [6] a universal domain is constructed: that is such a domain $D$ that any $G$ can be exhausted by it: the set $\mathrm{E}(D)$ in this case contains all classes of bounded domains of $\mathbb{C}^{n}(\# \mathrm{E}(D)=\infty)$.

If $D$ is a homogeneous domain, then $\# \mathrm{E}(D)=1$ : any $G$ that can be exhausted by $D$ is biholomorphically equivalent to $D$ (see [5]). A natural question is: if a bounded domain $D$ can exhaust only the domains that are biholomorphically equivalent to $D$, is $D$ necessarily homogeneous?

We present two results in this direction. First we present two kinds of counterexamples. The first kind are domains (see Theorem 1.1) that have non-smooth 
boundaries, and form an everywhere dense (in the Hausdorff metric) collection of domains. The second kind are domains (see Theorem 1.2) that are contractible, have piecewise smooth boundaries, but are not pseudoconvex. Later we give an affirmative answer to the question above for the case where the boundary $\partial D$ is twice continuously differentiable.

Theorem 1.1. Let $M$ be a bounded domain in $\mathbb{C}^{n}$. Then for any neighborhood of the boundary $U \supset \partial M$, there exists a countable set $S \subset U \cap M$ of isolated points such that $D=M \backslash S$ has the property $\# \mathrm{E}(D)=1$.

Theorem 1.2. For $n \geq 2$ there exists a contractible domain $\Omega$ with piecewise smooth boundary such that $\# E(\Omega)=1$ and $\Omega$ is not homogeneous.

In [5] it is proved that if a complete hyperbolic manifold $M$ can be exhausted by a bounded $C^{3}$ strictly pseudoconvex domain $D$ in $\mathbb{C}^{n}$, then $M$ is biholomorphic either to $D$ or to $B_{n}$. In light of this result, it is natural to ask whether every bounded $C^{3}$ strongly pseudoconvex domain can exhaust the ball. In this note we answer the question above in the affirmative. In fact we will prove a stronger statement:

Theorem 1.3. Let $D$ be a domain in $\mathbb{C}^{n}$ contained in a ball $U$ (of finite radius) such that $\partial D$ touches $\partial U$ at a point $p$ and $\partial D$ is $C^{2}$ in a neighborhood of $p$. Then $D$ can exhaust the unit ball in $\mathbb{C}^{n}$.

Remark. A seemingly possible approach to prove this theorem is to blow up $D$ at $p$ directly by Möbius transformations of $U$. This does not work for $n \geq 2$. (See the remark at the end of paper).

The following statements are direct consequences of Theorem 1.3.

Corollary 1.4. Every bounded domain in $\mathbb{C}^{n}$ with $C^{2}$ boundary can exhaust the unit ball in $\mathbb{C}^{n}$.

Corollary 1.5. If $\partial D$ is $C^{2}$ and $\# E(D)=1$, then $D \sim B_{n}$.

Corollary 1.6. Every bounded convex domain in $\mathbb{C}^{n}$ that has a $C^{2}$ strongly convex boundary point can exhaust the ball. So, if $D$ is a bounded convex domain in $\mathbb{C}^{n}$ that has a $C^{2}$ strongly convex boundary point and if $\# E(D)=1$, then $D \sim B_{n}$.

Corollary 1.7. Let $D$ be a region in $\mathbb{C}$ such that there exists a disk $V$ so that $V \cap \partial D$ is a Jordan arc. Then $D$ can exhaust the unit disk.

Remark. We are still unable to answer the following question for a nonconvex strictly pseudoconvex domain. Let $D$ be a such a domain and $p \in \partial D$. 
Does there exist an exhausting sequence $\left\{f_{j}\right\}$ from $D$ to the unit ball $B_{n}$ such that $f_{j}^{-1}(0) \rightarrow p$ ?

Acknowledgment. The authors would like to thank S.G. Krantz, who told them a conjecture of Kim and Cheung which brought their attention to the problems considered here. The second author would like to thank K.-T. Kim for a helpful conversation.

2. Proof of Theorems 1.1 and 1.2. In order to prove the theorems we will need several lemmas.

Lemma 2.1. Let $f_{k}: D \rightarrow G$ be an exhausting sequence. Suppose that for a point $w \in G,\left\{f_{k}^{-1}(w)\right\}$ b $D$ (that is, the closure of this sequence is compact in $D)$. Then $G$ is biholomorphically equivalent to $D$.

Proof. This follows from a normal family argument. Also, see [5].

We will need the definition of the Carathéodory metric on a complex manifold (see [1]). Let $\Delta$ be the unit disc in $\mathbb{C}$, and $P(\cdot, \cdot)$ the Poincaré distance on $\Delta$. Let $H(D, \Delta)$ denote the family of holomorphic maps from $D$ to $\Delta$. Then the Carathéodory distance $C_{D}(w, z)$ between $w$ and $z$ is defined by

$$
C_{D}(w, z)=\sup _{f \in H(D, \Delta)} P(f(w), f(z)) .
$$

It follows from the definition that $C_{D}$ is invariant under biholomorphic transformations, and that $C_{D}$ can be extended to the envelope of holomorphy of $D$. By $B_{D}^{C}(z, r)$ we denote the Carathéodory balls. The following two statements are direct consequences of the definition.

Lemma 2.2. Let $d, G$ be domains in $\mathbb{C}^{n}$, and $r>0$. Suppose that $z \in D \subset$ G. Then $B_{D}^{C}(z, r) \subset B_{G}^{C}(z, r)$.

Lemma 2.3. Let $D \subset M$ be bounded domains in $\mathbb{C}^{n}$ such that $M$ is contained in the envelope of holomorphy of $D$. Then $C_{D}(z, w)=C_{M}(z, w)$ for $z, w \in D$.

Definition 2.1. Let $D$ be a bounded domain in $\mathbb{C}^{n}$. Let $\mu: D \rightarrow(0, \infty]$ be defined by

$$
\mu(z)=\mu_{D}(z)=\sup \left\{r: B_{D}^{C}(z, r) \text { b } D\right\} .
$$


Then $\mu$ is invariant under biholomorphic mappings. If a boundary point $p$ of $D$ lies in the envelope of $D$, then $\lim _{z \rightarrow p} \mu(z)=0$.

Lemma 2.4. Let $D$ be a bounded domain in $\mathbb{C}^{n}$ such that

$$
\lim _{z \rightarrow \partial D} \mu_{D}(z)=0
$$

Then $\# E(D)=1$.

Proof. Suppose that a bounded domain $G$ can be exhausted by $D$ and $f_{k}: D \rightarrow G$ is an exhausting sequence. Pick a point $z \in G$ and $r>0$ such that $B_{G}^{C}(z, 2 r) \mathrm{b} G$. Then for large enough $k, B_{G}^{C}(z, r) \mathrm{b} f_{k}(D)$, hence $B_{f_{k}(D)}^{C}(z, r) \subset$ $B_{G}^{C}(z, r)$ b $f_{k}(D)$ by Lemma 2.2. Therefore $r \leq \mu_{f_{k}(D)}(z)=\mu_{D}\left(f_{k}^{-1}(z)\right)$. This, together with (2.1), implies that $\left\{f_{k}^{-1}(z)\right\}$ b $D$. By Lemma 2.1, $G$ is biholomorphically equivalent to $D$.

Proof of Theorem 1.1. Pick $S=\left\{z_{k}\right\}$ as a sequence of isolated points in $U \cap M$ such that: $\bar{S} \cap M=S$ and for any $r>0$ there exists an open neighborhood $V_{r} \supset \partial M$ such that if $z \in V_{r} \cap M$, then $B_{M}^{C}(z, r) \cap S \neq \varnothing$. Clearly there are many ways to construct such a sequence. Let $D=M \backslash S$. It follows that (2.1) holds for $D$. By Lemma 2.4, \#E(D)=1.

Now let $\Delta^{n}$ denote the polydisc in $\mathbb{C}^{n}$. For $z \in \mathbb{C}^{n}$ let

$$
\begin{aligned}
M(z) & =\max \left(\left|z_{1}\right|, \ldots,\left|z_{n}\right|\right) \\
m(z) & =\min \left(\left|z_{1}\right|, \ldots,\left|z_{n}\right|\right), \\
a(z) & =(1 / n)\left(\left|z_{1}\right|^{2}+\cdots+\left|z_{n}\right|^{2}\right) .
\end{aligned}
$$

Let

$$
\begin{aligned}
& D_{1}=\left\{z \in \Delta^{n}: M(z)-m(z)<(1-M(z))^{2}\right\} \\
& D_{2}=\left\{z \in \Delta^{n}:\left(\left|z_{1}\right|^{2}-a(z)\right)^{2}+\cdots+\left(\left|z_{n}\right|^{2}-a(z)\right)^{2}<(1-a(z))^{4}\right\} .
\end{aligned}
$$

Then both $D_{1}$ and $D_{2}$ are Reinhardt domains with piecewise smooth boundary, they both have $\Delta^{n}$ as envelope of holomorphy, and $T^{n} \equiv\left\{z \in \mathbb{C}^{n}:\left|z_{1}\right|=\cdots=\right.$ $\left.\left|z_{n}\right|=1\right\}$ as distinguished boundary. The boundary of $D_{2}$ is "smoother" than that of $D_{1}$.

Lemma 2.5. The domains $D_{1}$ and $D_{2}$ satisfy (2.1). 
Proof. The proofs for $D_{1}$ and $D_{2}$ are similar, but the proof for $D_{2}$ needs more computations. So we will only prove $(2.1)$ for $D \equiv D_{1}$. The envelope of holomorphy of $D$ is $\Delta^{n}$. If $p \in \partial D \backslash T^{n}$, then $p$ lies in that envelope, hence $\lim _{z \rightarrow p} \mu(z)=0$.

So we only need to consider the points near $T^{n}$. We now estimate $\mu_{D}(z)$ for a $z=\left(z_{1}, \ldots, z_{n}\right) \in D$ near $T^{n}$. Without loss of generality we may assume $z_{1} \geq$ $z_{2} \geq \cdots \geq z_{n}>1 / 2$. Then $z_{1}=M(z)$ and $z_{n}=m(z)$. Let $u=z_{1}-\left(1-z_{1}\right)^{2}$, and $q=\left(z_{1}, \ldots, z_{n-1}, u\right)$. Then $u<z_{n} \leq z_{1}$ and $q \in \partial D$. Thus

$$
\begin{aligned}
\mu_{D}(z) & \leq C_{D}(z, q)=P\left(z_{n}, u\right) \leq P\left(z_{1}, u\right) \\
& =2 \int_{u}^{z_{1}} \frac{d t}{1-t^{2}} \leq 2 \frac{z_{1}-u}{1-z_{1}^{2}} \\
& =2 \frac{\left(1-z_{1}\right)^{2}}{1-z_{1}^{2}} \leq 2\left(1-z_{1}\right)=2(1-M(z)) .
\end{aligned}
$$

Therefore $\lim _{z \in D, z \rightarrow T^{n}} \mu_{D}(z)=0$.

Proof of Theorem 1.2. It follows from the construction that the domains $D_{1}$ and $D_{2}$ are contractible with piecewise smooth boundaries. By Lemmas 2.4 and 2.5 , they satisfy $\# E\left(D_{1}\right)=1$ and $\# E\left(D_{2}\right)=1$. Since they are not pseudoconvex, they are not homogeneous.

3. Proof of Theorem 1.3. We now consider $n \geq 2$. For $n=1$, see the remark after the proof. We first introduce some notation and terminology. We write the coordinates of $\mathbb{C}^{n}$ as $z=\left(z_{1}, z^{\prime}\right), z^{\prime}=\left(z_{2}, \ldots, z_{n}\right)$. Let

$$
\begin{aligned}
q_{k}(z) & =2 \operatorname{Re} z_{1}+\left|z_{1}\right|^{2}+k\left|z^{\prime}\right|^{2}, \quad k \in \mathbb{N} \\
Q_{k} & =\left\{z \in \mathbb{C}^{n}: q_{k}(z)<0\right\}, \quad k \in \mathbb{N}, \\
B & =Q_{1}, \\
\alpha B & =\left\{z:\left|z_{1}+\alpha\right|^{2}+\left|z^{\prime}\right|^{2}<\alpha^{2}\right\}, \\
B(r) & =\left\{z \in \mathbb{C}^{n}:\left|z_{1}+2\right|^{2}+\left|z^{\prime}\right|^{2}<r^{2}\right\}, \\
S & =Q_{3} \cup B(1 / 8), \\
T & =Q_{2} \cup B(1 / 7) .
\end{aligned}
$$

Clearly, $Q_{k+1} \subset Q_{k}$, and all $Q_{k}$ 's are biholomorphic to the unit ball. $B \equiv Q_{1}$ is the unit ball centered at $\left(-1,0^{\prime}\right)$.

Definition 3.1. Let $N, k \in \mathbb{Z}, N \geq 4,0 \leq k \leq 2(N-4)$. A domain $D \subset \mathbb{C}^{n}$ is said to be a domain of type $C(N, k)$ if $D$ satisfies the following conditions: 
(a) $D \subset B$, and $0 \in \partial D$;

(b) $\partial D$ is $C^{2}$ near 0 ;

(c) There is a $C^{2}$ defining function of $D$ at 0 of the form

$$
\rho(z)=2 \operatorname{Re} z_{1}+\operatorname{Re} \sum_{i, j=2}^{n} a_{i j} z_{i} z_{j}+d\left|z_{1}\right|^{2}+N\left|z^{\prime}\right|^{2}+o\left(|z|^{2}\right),
$$

where the numbers $N, d$ and $a_{i j}(i, j \geq 2)$ satisfy

$$
d>1,
$$

$$
\operatorname{Re} \sum_{i, j=2}^{n} a_{i j} z_{i} z_{j} \quad+(N-4-k / 2)\left|z^{\prime}\right|^{2} \geq 0, \quad z^{\prime} \in \mathbb{C}^{n-1} .
$$

Note that (3.2) implies that

$$
\left|\sum_{i, j=2}^{n} a_{i j} z_{i} z_{j}\right| \leq(N-4-k / 2)\left|z^{\prime}\right|^{2}, \quad z^{\prime} \in \mathbb{C}^{n-1} .
$$

Consider the maps $f_{\varepsilon}: B \rightarrow B$ defined by

$$
\begin{aligned}
w_{1} & =\frac{\varepsilon z_{1}}{2-\varepsilon+(1-\varepsilon) z_{1}} \\
w^{\prime} & =\frac{\sqrt{\varepsilon(2-\varepsilon)}}{2-\varepsilon+(1-\varepsilon) z_{1}} z^{\prime} .
\end{aligned}
$$

The $f_{\varepsilon}$ 's are Möbius transformations of $B$. One can check that $f_{\varepsilon}\left(Q_{k}\right)=Q_{k}$.

We will also consider the entire analytic map $G_{b}$ defined by $G_{b}(z)=\left(z_{1}+\right.$ $\left.\sum_{j=1}^{n} b_{j} z_{1} z_{j}, z^{\prime}\right)$, where the $b_{j}$ 's are complex numbers.

Lemma 3.1. There exists $a \lambda>0$ such that if $b_{j}$ are complex numbers that satisfy

$$
\sum_{j=1}^{n}\left|b_{j}\right| \leq \lambda
$$

then $G_{b}$ is an injective holomorphic map in a neighborhood of $\bar{B}$, and $G_{b}(B) \subset$ $2 B$.

Proof. By direct computations. 
Lemma 3.2. Let $D$ be a domain as in Theorem 1.3. Then there is an integer $N \geq 4$ such that $D$ is biholomorphic to a domain of type $C(N, 0)$.

Proof. Without loss of generality we may assume that $D \subset B$ and $0 \in \partial D$. After a unitary transformation in the $z^{\prime}$ directions, the defining function of $D$ at 0 can be written as

$$
\begin{aligned}
\rho(z)=2 \operatorname{Re} z_{1} & +\operatorname{Re} \sum_{i, j=1}^{n} a_{i j} z_{i} z_{j} \\
& +\operatorname{Re} \sum_{j=1}^{n} c_{j} z_{1} \bar{z}_{j}+\sum_{j=2}^{n} N_{j}\left|z_{j}\right|^{2}+o\left(|z|^{2}\right) .
\end{aligned}
$$

The fact that $D \subset B$ implies that

$$
\operatorname{Re} \sum_{i, j=2}^{n} a_{i j} z_{i} z_{j}+\sum_{j=2}^{n} N_{j}\left|z_{j}\right|^{2} \geq\left|z^{\prime}\right|^{2}, \quad z^{\prime} \in \mathbb{C}^{n-1} .
$$

Thus each $N_{j} \geq 1$.

Consider a biholomorphic map $H: \mathbb{C}^{n} \rightarrow \mathbb{C}^{n}$ defined by

$$
H_{1}(z)=z_{1}, \quad H_{j}(z)=z_{j}+\left(c_{j} /\left(2 N_{j}\right)\right) z_{1}, \quad j=2, \ldots, n .
$$

The defining function $\rho \circ H^{-1}$ of the domain $D^{\prime} \equiv H(D)$ at 0 again has the form (3.6), but with $c_{j}=0$ for $j=2, \ldots, n$. The domain $D^{\prime}$ satisfies $D^{\prime} \subset\{z$ : $\left.\operatorname{Re} z_{1}<0\right\}, \partial D^{\prime} \cap\left\{z: \operatorname{Re} z_{1}=0\right\}=\{0\}$, and $\partial D^{\prime}$ is strongly convex near 0 . So, $D^{\prime}$ is contained in a big ball $\alpha B$. Shrinking $D^{\prime}$ in all directions and rescaling it in the $z^{\prime}$ directions, we obtain a domain $\tilde{D}$ that is biholomorphic to $D$, that is contained in $B$, and that has a defining function of the form

$$
\begin{aligned}
\tilde{\rho}(z)= & 2 \operatorname{Re} z_{1}+2 \operatorname{Re} \sum_{j=1}^{n} b_{j} z_{1} z_{j} \\
& +\operatorname{Re} \sum_{i, j=2}^{n} a_{i j} z_{i} z_{j}+d\left|z_{1}\right|^{2}+M\left|z^{\prime}\right|^{2}+o\left(|z|^{2}\right) .
\end{aligned}
$$

Consider the domain $f_{\varepsilon}^{-1}(\tilde{D}) \subset B$, whose defining function can be chosen as

$$
\rho_{\varepsilon}(z)=\frac{\left|2-\varepsilon+(1-\varepsilon) z_{1}\right|^{2}}{\varepsilon(2-\varepsilon)}\left(\tilde{\rho} \circ f_{\varepsilon}(z)\right) .
$$


One can check that $\rho_{\varepsilon}$ again has the form (3.7), but the coefficients are changed to

$$
a_{i j}^{\prime}=a_{i j} ; \quad b^{\prime}{ }_{j}=\sqrt{\frac{\varepsilon}{2-\varepsilon}} b_{j},(j \geq 2) ; \quad b_{1}^{\prime}=\frac{\varepsilon}{2-\varepsilon} b_{1} ; \quad d^{\prime}=1+\frac{\varepsilon}{2-\varepsilon}(d-1) .
$$

Thus, considering $f_{\varepsilon}^{-1}(\tilde{D})$ for a sufficiently small $\varepsilon>0$ in place of $\tilde{D}$, if necessary, we can assume that the numbers $b_{j}$ in (3.7) satisfy (3.5). By Lemma $3.1, \hat{D} \equiv$ $G_{b}(\tilde{D}) \subset 2 B$. One can check that the defining function $\hat{\rho} \equiv \tilde{\rho} \circ G_{b}^{-1}$ of $\hat{D}$ has the form

$$
\hat{\rho}(z)=2 \operatorname{Re} z_{1}+\operatorname{Re} \sum_{i, j=2}^{n} a_{i j} z_{i} z_{j}+d\left|z_{1}\right|^{2}+M\left|z^{\prime}\right|^{2}+o\left(\left|z^{\prime}\right|^{2}\right) .
$$

Choose an integer $N>8 M$, and let $\beta=M / N$. Then $\beta<1 / 8$. Let $D_{1}=\beta \hat{D}$. Then $D_{1} \subset(1 / 4) B$. The defining function $\beta \hat{\rho}(z / \beta)$ of $D_{1}$ has the form (3.1) (with the $a_{i j}$ 's, $d$ different from those in $\left.\hat{\rho}(z)\right)$. Now the defining function of (1/4)B can be chosen as $h(z)=2 \operatorname{Re} z_{1}+4|z|^{2}$. The fact that $D_{1} \subset(1 / 4) B$ implies (3.2) with $k=0$. Thus $D_{1}$ is a domain of type $C(N, 0)$.

For complex numbers $b_{i j}, 2 \leq i, j \leq n$, we define a biholomorphic mapping $F_{b}: \mathbb{C}^{n} \rightarrow \mathbb{C}^{n}$ by

$$
F_{b}\left(z_{1}, z^{\prime}\right)=\left(z_{1}+\sum_{i, j=2}^{n} b_{i j} z_{i} z_{j}, z^{\prime}\right) .
$$

Lemma 3.3. Let $b_{i j}, 2 \leq i, j \leq n$, be complex numbers satisfying

$$
\left|\sum_{i, j=2}^{n} b_{i j} z_{i} z_{j}\right| \leq \frac{1}{4}\left|z^{\prime}\right|^{2}, \quad z^{\prime} \in \mathbb{C}^{n-1} .
$$

Then $F_{b}(S) \subset T$.

Proof. It is clear that $F_{b}(B(1 / 8)) \subset B(1 / 7)$. So we only need to show that $F_{b}\left(Q_{3}\right) \subset Q_{2}$, which is equivalent to $q_{2} \circ F_{b}(z) \leq 0$ when $z \in \partial Q_{3}$. For $z \in \partial Q_{3}$, we have $q_{3}(z)=0,\left|z_{1}+1\right| \leq 1,\left|z^{\prime}\right|^{2} \leq 1 / 3$, hence

$$
\begin{aligned}
q_{2} \circ F_{b}(z) & =q_{2} \circ F_{b}(z)-q_{3}(z) \\
& =\left|\sum_{i, j=2}^{n} b_{i j} z_{i} z_{j}\right|^{2}+2 \operatorname{Re}\left[\left(\bar{z}_{1}+1\right) \sum_{i, j=2}^{n} b_{i j} z_{i} z_{j}\right]-\left|z^{\prime}\right|^{2} \\
& \leq(1 / 16)(1 / 3)\left|z^{\prime}\right|^{2}+(1 / 2)\left|z^{\prime}\right|^{2}-\left|z^{\prime}\right|^{2} \\
& \leq 0 .
\end{aligned}
$$

This completes the proof. 
Lemma 3.4. Suppose that $N \geq 5,0 \leq k \leq 2(N-4)-1$. Then every domain $D$ of type $C(N, k)$ is biholomorphic to a domain of type $C(N, k+1)$.

Proof. By definition, $D$ has a defining function of the form (3.1), where the numbers $a_{i j}(i, j \geq 2), d$, and $N$ satisfy (3.2). The inequality (3.2) implies that for $z$ sufficiently close to 0 and $z \in \partial Q_{3}, \rho(z) \geq 0$. Thus there exists an $\eta>0$ such that $D \cap\left\{z \in B: \operatorname{Re} z_{1} \geq-\eta\right\} \subset Q_{3}$. Choose an $\varepsilon>0$ so small that $f_{\varepsilon}^{-1}\left(\left\{z \in B: \operatorname{Re} z_{1} \leq-\eta\right\}\right) \subset B(1 / 8)$. Since

$$
f_{\varepsilon}^{-1}\left(D \cap\left\{z \in B: \operatorname{Re} z_{1} \geq-\eta\right\}\right) \subset f_{\varepsilon}^{-1}\left(Q_{3}\right)=Q_{3},
$$

we have $f_{\varepsilon}^{-1}(D) \subset S$.

Let

$$
b_{i j}=\frac{1}{4(N-4-k / 2)} a_{i j}, \quad 2 \leq i, j \leq n .
$$

Then the numbers $b_{i j}$ satisfy (3.9). By Lemma 3.3, $F_{b}(S) \subset T$.

Let $h\left(z_{1}, z^{\prime}\right)=\left((14 / 15) z_{1}, \sqrt{14 / 15} z^{\prime}\right)$. One can check that $h(T) \subset B$. Therefore $\hat{D} \equiv h \circ F_{b} \circ f_{\varepsilon}^{-1}(D) \subset B$. One can check that the defining function of $\hat{D}$ near the origin has the form

$$
\begin{aligned}
\hat{\rho}(z) & =\frac{14}{15} \frac{\left|2-\varepsilon+15(1-\varepsilon) z_{1} / 14\right|^{2}}{\varepsilon(2-\varepsilon)} \rho \circ f_{\varepsilon} \circ F_{b}^{-1} \circ h^{-1}(z) \\
& =2 \operatorname{Re} z_{1}+\operatorname{Re} \sum_{i, j=2}^{n}\left(a_{i j}-2 b_{i j}\right) z_{i} z_{j}+d^{\prime}\left|z_{1}\right|^{2}+N\left|z^{\prime}\right|^{2}+o\left(|z|^{2}\right),
\end{aligned}
$$

where

$$
d^{\prime}=\frac{15}{14}\left[1+\frac{\varepsilon}{2-\varepsilon}(d-1)\right]>1
$$

Since

$$
a_{i j}-2 b_{i j}=\frac{N-4-(k+1) / 2}{N-4-k / 2} a_{i j},
$$

we have

$$
\left|\sum_{i, j=2}^{n}\left(a_{i j}-2 b_{i j}\right) z_{i} z_{j}\right| \leq(N-4-(k+1) / 2)\left|z^{\prime}\right|^{2}, \quad z^{\prime} \in \mathbb{C}^{n-1} .
$$

Therefore $\hat{D}$ is a domain of type $C(N, k+1)$. 
Lemma 3.5. Let $D$ and $G$ be bounded domains in $\mathbb{C}^{n}$. Suppose that $G$ is starlike with respect to $p \in G$. If there is a sequence $\left\{h_{k}\right\}$ of injective holomorphic maps from $D$ to $\mathbb{C}^{n}$ such that $h_{k}(D) \rightarrow G$ in the Hausdorff metric, then $D$ can exhaust $G$.

Proof. We may assume that $p=0$. Let $K \mathrm{~b} G$. Choose $\varepsilon>0$ such that $[(1+\varepsilon) /(1-\varepsilon)] K \subset G$. Then choose $k$ such that $(1-\varepsilon) G \subset h_{k}(D) \subset(1+\varepsilon) G$. Let $g=(1+\varepsilon)^{-1} h_{k}$. Then $K \subset g(D) \subset G$.

Proof of Theorem 1.3. By Lemma 3.2, we can assume that the domain $D$ is of type $C(N, 0)$. Recall that $N \geq 4$. If $N \geq 5$, then by Lemma 3.4 and induction, $D$ is biholomorphic to a domain of type $C(N, 2(N-4)$ ). (If $N=4$, then $C(N, 0)$ is the same as $C(N, 2(N-4)))$. The inequality (3.3) with $k=2(N-4)$ forces $\sum a_{i j} z_{i} z_{j}=0$. So, there exists a biholomorphic image $D_{1} \subset B$ of $D$ that has a defining function of the form

$$
\rho_{1}(z)=2 \operatorname{Re} z_{1}+d\left|z_{1}\right|^{2}+N\left|z^{\prime}\right|^{2}+o\left(|z|^{2}\right) .
$$

Direct computations give that

$$
\lim _{\varepsilon \rightarrow 0}\left[\frac{\left|2-\varepsilon+(1-\varepsilon) z_{1}\right|^{2}}{\varepsilon(2-\varepsilon)} \rho_{1} \circ f_{\varepsilon}(z)\right]=q_{N}(z) .
$$

Thus $f_{\varepsilon}^{-1}\left(D_{1}\right) \rightarrow Q_{N}$ in the Hausdorff metric. Since $Q_{N}$ is convex and therefore starlike, by Lemma 3.5, $D_{1}$ can exhaust $Q_{N}$. Hence $D$ can exhaust the ball. This completes the proof.

Remark. A direct application of Möbius transformations (3.4) does not work for $n \geq 2$, the reason being that second order terms of the form $\sum a_{i j} z_{i} z_{j}$ would not be eliminated this way. However in case $n=1$ (since those terms are not present) it produces the desired result.

\section{REFERENCES}

[1] C. CARAthÉodory, Über die abbildungen, die durch systeme von analytischen funktionen von mehreren veranderlichen erzeugt werden. Math. Z. 34 (1932), 758-792.

[2] C. K. Cheung, On some properties of exhaustion maps between bounded domains. Pacific J. Math. 156 (1992), 107-117.

[3] J. E. Forness and E. L. Stout, Polydiscs in complex manifolds. Math. Ann. 227 (1977), 145-153. 
[4] J. E. Fornæss And N. Sibony, Increasing sequences of complex manifolds. Math. Ann. 255 (1981), 351-360.

[5] B. L. Fridman, Biholomorphic invariants of a hyperbolic manifold and some applications. Trans. Amer. Math. Soc. 276 (1983), 685-698.

[6] B. L. Fridman, A universal exhausting domain. Proc. Amer. Math. Soc. 98 (1986), 267-270.

[7] A. Kodama, S. G. Krantz and D. Ma, A characterization of generalized complex ellipsoids in $\mathbb{C}^{n}$ and related problems. Indiana Univ. Math. J. 41 (1992), 173-195.

Buma Fridman

Department of Mathematics and Statistics

Wichita State University

Wichita, Kansas 67260-0033

E-MAIL: fridman@twsuvm.uc.twsu.edu

DAwor MA

Department of Mathematics and Statistics

Wichita State University

Wichita, KS 67260-0033

E-maIL: dma@cs.twsu.edu

Received: May 9th, 1994; revised: November 29th, 1994. 